\title{
Epidermoid splenic cyst with elevated serum level of CAig-9
}

\author{
Natalia Buda ${ }^{1}$, Anna Wszołek² ${ }^{2}$ Maciej Śledziński³, Anton Żawrocki ${ }^{4}$, and Krzysztof Sworczak ${ }^{2}$
}

Departments of ${ }^{1}$ Internal Medicine, Connective Tissue Diseases and Geriatrics, ${ }^{2}$ Endocrinology and Internal Medicine, ${ }^{3}$ General, Endocrine and Transplant Surgery, and ${ }^{4}$ Pathology, Medical University of Gdansk, Gdansk, Poland

Received: January 7, 2017

Revised : May 1, 2017

Accepted: May 30, 2017

\section{Correspondence to}

Natalia Buda, Ph.D.

Tel: +48-600369310

Fax: +48-600369310

E-mail: natabud@wp.pl
The 25-year old patient, was hospitalized due to the abdomen pain in the left upper quadrant, accompanied by watery diarrhoea, nausea, vomits, periodically occurring fever up to $39^{\circ} \mathrm{C}$ degrees with shivers and the body weight loss (15 kg during 2 months). There was conducted the extensive laboratory diagnostics and there was noted the high level of tumour markers in the plasma: cancer antigen 19.9 (CA19.9) 2,878 $\mathrm{U} / \mathrm{mL}(\mathrm{N}<37 \mathrm{U} / \mathrm{mL})$ and $\mathrm{CA}-12595 \mathrm{U} /$ $\mathrm{mL}(\mathrm{N}<35 \mathrm{U} / \mathrm{mL})$. The results of several tests performed on fecal for par- asite eggs and immunological tests of blood infection with hydit (Elis's and Western blot's methods) were negative. Within the diagnostic imaging was performed the ultrasound test of the abdomen, a static scintigraphy of the spleen and the liver with sulphidicid colloid-99m Tc + single-photon emission computed tomography/computed tomography (CT), magnetic resonance of the abdomen and the lesser pelvic with giving the contrast agent (Fig. 1). The ultrasonography (US) revealed typical splenic cysts' features: the complex
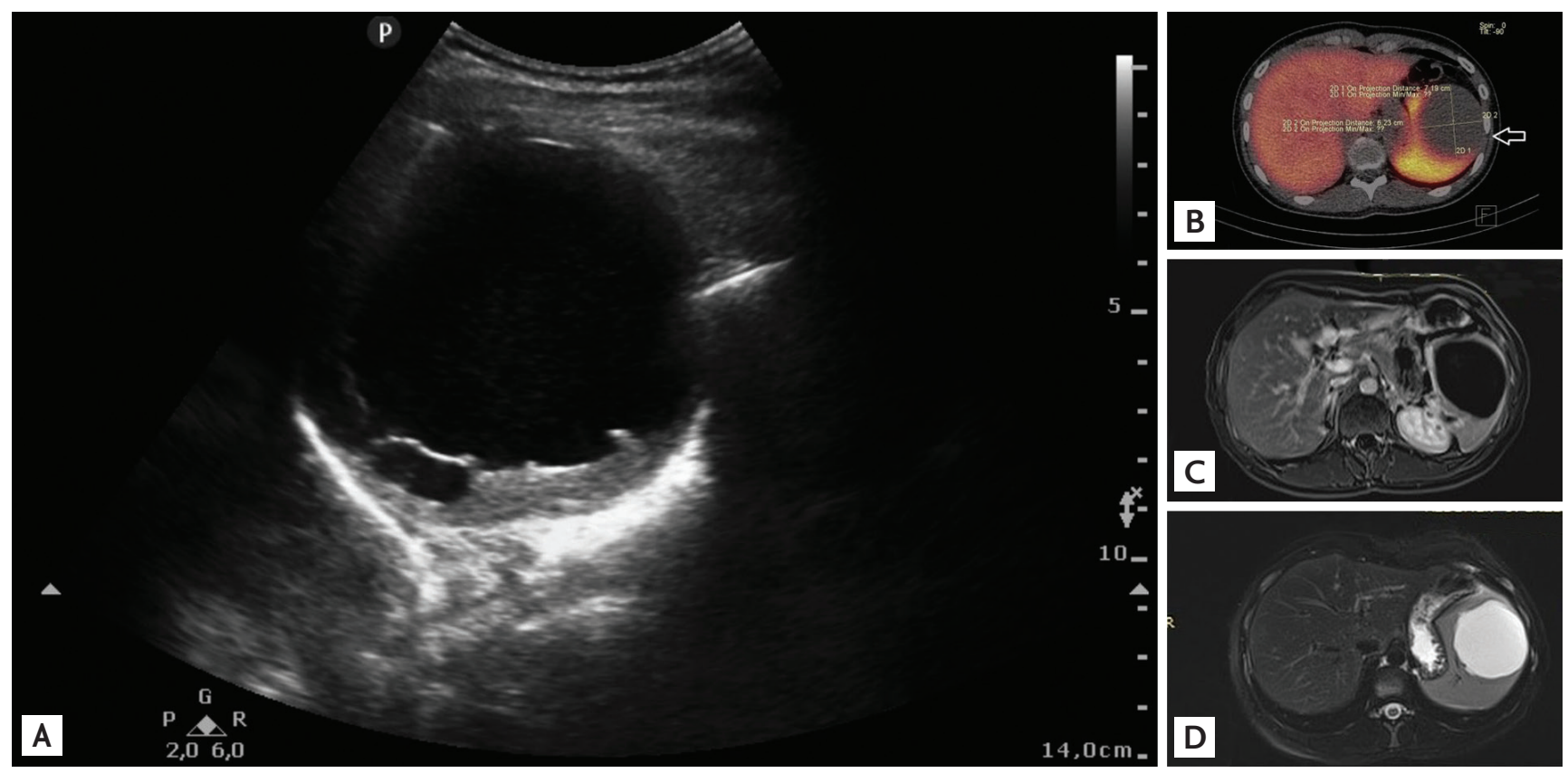

Figure 1. Imaging diagnostic tests. (A) Ultrasound examination of abdomen shows: anechoic, well demarcated, and capsulated spleen cyst. (B) Static scintigraphy of the liver and spleen in the lesion with sulphidic colloid- ${ }^{99 m} \mathrm{Tc}+$ single-photon emission computed tomography/computed tomography: there is no uptake of the marked sulphidic colloid. An arrow shows spleen cyst. (C, D) Magnetic resonance imaging of abdomen shows: hypointense cyst T1-weighted image and hyperintense T2-weighted image of the spleen. 

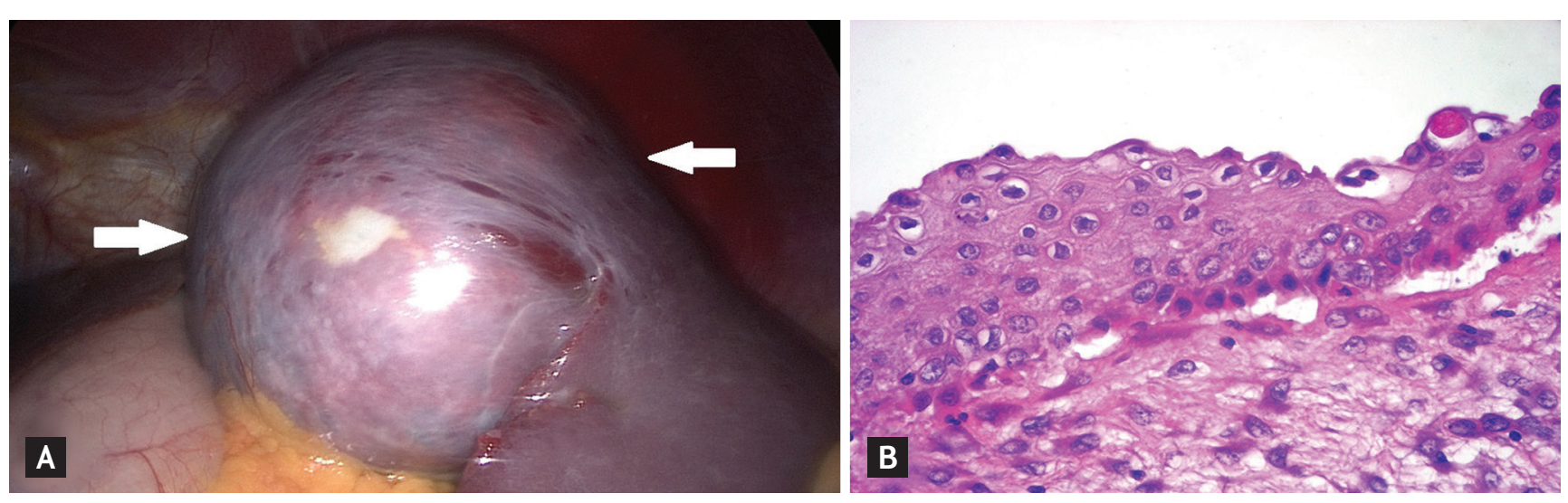

Figure 2. (A) Intraoperative image of the spleen cyst (arrows). (B) Histopathology: a cyst wall composed lined by thin multilayered nonkeratinizing squamous epithelium. Epidermoid splenic cyst $(\times 40)$.

of homogeneous, anechoic, well separated cysts, with the largest of $90 \times 80 \mathrm{~mm}$, divided with a septum into two chambers. One was filled with homogenous hyperechoic content. In the magnetic resonance imaging (MRI) the cysts were: hypointense on T1-weighted images and hyperintense on T2-weighted images and intensity of the signal was similar to water and with no contrast uptake.

Complaints reported by the patient, weight loss and large sizes and unclear character of the splenic cysts qualified for the laparoscopic splenectomy. The liquid culture from the cysts was negative. A histopathology indicated an epidermoid cyst. A cyst wall composed of a thick layer of fibrous tissue was partly lined by thin multilayered nonkeratinizing squamous epithelium; squamous differentiation was confirmed by strong diffuse cytoplasmic expression of $\mathrm{CK}_{5} / 6$ (Fig. 2). The postoperative course was not complicated. During the laboratory control ( 3 weeks after the surgery) was stated the normalization if the CA-125 level and significant $(\times 20)$ lowering of the CA19.9 level in plasma.

Coexistence of the increased CA19-9 and CA-125 in plasma with the epidermoid splenic cyst seems to be substantial in the differential diagnostics of the spleen lesions. In the diagnostics are also useful imaging tests like: the US and next CT or MRI. However the essential meaning has the histopathological test's result. In the case of the symptomatic cysts and of the large size $(>5 \mathrm{~cm})$, the chosen treatment is the partial or complete splenectomy.

\section{Conflict of interest}

No potential conflict of interest relevant to this article was reported. 